\title{
Inability to access addiction treatment predicts injection initiation among street- involved youth in a Canadian setting
}

\author{
Kora DeBeck ${ }^{1,2^{*}}$, Thomas Kerr ${ }^{1,3}$, Seonaid Nolan ${ }^{1,3}$, Huiru Dong ${ }^{1}$, Julio Montaner ${ }^{1,3}$ and Evan Wood ${ }^{1,3}$
}

\begin{abstract}
Background: Preventing injection drug use among vulnerable youth is critical for reducing serious drug-related harms. Addiction treatment is one evidence-based intervention to decrease problematic substance use; however, youth frequently report being unable to access treatment services and the impact of this on drug use trajectories remains largely unexplored. This study examines the relationship between being unable to access addiction treatment and injection initiation among street-involved youth.

Methods: Data were derived from the At-Risk Youth Study (ARYS), a prospective cohort of street-involved youth aged 14-26 who use illicit drugs, from September 2005 to May 2014. An extended Cox model with time-dependent variables was used to identify factors independently associated with injection initiation.

Results: Among 462 participants who were injection naive at baseline, 97 (21\%) initiated injection drug use over study follow-up and 129 (28\%) reported trying but being unable to access addiction treatment in the previous 6 months at some point during the study period. The most frequently reported reason for being unable to access treatment was being put on a wait list. In a multivariable Cox regression analysis, being unable to access addiction treatment remained independently associated with a more rapid rate of injection initiation (Adjusted Hazard Ratio $=2.02 ; 95 \%$ Confidence Interval: 1.12-3.62), after adjusting for potential confounders.

Conclusion: Inability to access addiction treatment was common among our sample and associated with injection initiation. Findings highlight the need for easily accessible, evidence-based addiction treatment for high-risk youth as a means to prevent injection initiation and subsequent serious drug-related harms.
\end{abstract}

Keywords: Injection initiation, At-risk youth, Addiction treatment, Injection prevention

\section{Findings}

Preventing vulnerable youth from initiating injection drug use is critical for reducing drug-related morbidity and mortality [1-4]. There are a number of features of young drug injectors that highlight the urgency of intervening early in their drug use trajectories to prevent the transition to injection drug use [5]. For instance, prior research among street-involved youth indicates that once youth initiate injection drug use, the majority rapidly become established injectors [6]. Young new injection initiators are also more prone to engage in risky drug

\footnotetext{
* Correspondence: urhi-kd@cfenet.ubc.ca

'British Columbia Centre for Excellence in HIV/AIDS, Vancouver, Canada

${ }^{2}$ School of Public Policy, Simon Fraser University, Vancouver, Canada

Full list of author information is available at the end of the article
}

use practices that put them at higher risk of drug overdose and infectious disease transmission [3, 4, 7-9].

Structural level influences, such as homelessness and unemployment [10-12], alongside individual level factors including childhood trauma, and specific drug use patterns $[13,14]$, have been recognized as factors that facilitate transitions into injection drug use among vulnerable youth. While these findings suggest that injection prevention efforts should be directed to the areas of housing, employment, and childhood trauma prevention and recovery, addiction treatment may provide additional opportunities to reduce injection initiation. It has long been established that addiction treatment is one of the most cost-effective interventions to reduce problematic substance use $[15,16]$. However, prior studies 
indicate that many vulnerable individuals are unable to access addiction treatment [17-20]. To determine the role that barriers to accessing addiction treatment may play in influencing drug use trajectories, we examined whether inability to access addiction treatment was associated with injection initiation among a cohort of streetinvolved youth.

\section{Methods}

Data for this study was obtained from the At-Risk Youth Study (ARYS), which is an open prospective cohort of street-involved youth in Vancouver, Canada that has been described in detail previously [21]. In brief, study recruitment is open and undertaken using snowball sampling and extensive street-based outreach methods. To be eligible, participants at recruitment must be age 14-26 years, have used illicit drugs in the past 30 days, and provide written informed consent. At baseline and on a semi-annual basis, participants complete an interviewer-administered questionnaire that elicits information related to drug use and contact with health and social services. At each study visit participants are provided with a stipend (\$30 Canadian currency) for their time. The study has been approved by the University of British Columbia's Research Ethics Board.

The study period for this analysis was September 2005 to May 2014. To examine the potential relationship between initiation into injection drug use and inability to access addiction treatment, all participants who had never injected drugs at baseline and had completed at least one follow-up visit during the study period were included in the present analysis. The primary outcome of interest was injection initiation which was defined as the midpoint between the last report of remaining injection naive and the first report of having used a needle to chip, fix or muscle drugs. For descriptive purposes we also assessed the median number of years between initiation of non-injection "hard" drug use (defined as use of heroin, cocaine, crack, or crystal methamphetamine) and initiation of injection drug use. These estimates were based on the reported age of first non-injection "hard" drug use, and age of participants at the midpoint between the last report of remaining injection naïve and the first report of having used a needle to chip, fix, or muscle drugs. The primary explanatory variable of interest was being unable to access addiction treatment defined as responding affirmatively to the question: "In the past 6 months, have you tried to access any treatment program but were unable?" Participants were also asked to specify the types of addiction treatment they had difficulty accessing (e.g., detox, recovery house, treatment center, counselor, other), as well as the main reason they were unable to access the program (waiting lists, behavioral issues, rejection from program, logistics such as hours of optional, location, paperwork etc.).

To determine whether there was a significant relationship between our outcome of interest and our primary explanatory variable we a priori selected a range of secondary explanatory variables we hypothesized might be associated with both injection initiation and being unable to access addiction treatment. Secondary explanatory factors included: number of years since initiated "hard" drug use defined as use of cocaine, crack, heroin, or crystal methamphetamine (per additional year); gender (female vs. male); ethnicity (Caucasian vs. other); non-injection cocaine use (yes vs. no); crack smoking (yes vs. no); non-injection crystal methamphetamine use (yes vs. no); and non-injection heroin use (yes vs. no). All drug use variables including being unable to access addiction treatment refer to circumstances and behaviors over the previous 6 months and were treated as time-updated covariates on the basis of semi annual follow-up data. In addition, to protect against reverse causation whereby reported behaviors were a consequence of drug injecting, all drug use variables including being unable to access addiction treatment were lagged to the previous available observation $[10,11]$.

To assess the relationship between being unable to access addiction treatment and injection initiation, as a first step we calculated the incidence density of injection initiation using a Poisson model. Then, using an extended Cox model with time-dependent variables, we estimated the unadjusted relative hazards and $95 \%$ confidence intervals for factors associated with injection initiation [22]. To fit our multivariable Cox model, we ran a fixed multivariable model where all variables of interest were included into a single model. All statistical analyses were performed using SAS software version 9.3 (SAS, Cary, NC, USA). All tests of significance were two-sided.

\section{Results}

Overall, 1157 street-involved youth were recruited into the ARYS cohort during the study period. At enrolment 659 (57 \%) youth had never injected drugs. Among this group, during the study period, the average yearly loss to follow-up rate was $3.15 \%$. At the time the analysis was conducted, 462 (70 \%) participants completed at least one study follow-up to assess for injection initiation and were therefore included in the analysis. There were no significant differences with respect to gender (Chi-square $p$-value $=0.943$; degrees of freedom $[\mathrm{df}]=1$ ) or ethnicity (Chi-square $p$-value $=0.117 ; \mathrm{df}=1$ ) between the 462 youth who represented the eligible study population and the 197 injecting naïve youth who were ineligible because they either did not have a follow up visit at the time the analysis 
was conducted or were not enrolled in the cohort long enough to be due for a study follow-up.

Among the sample of 462 youth included in the study, 142 (31 \%) were female and the median age was 21.5 years (interquartile range $[\mathrm{IQR}]=19.6-23.2$ ). The median number of study visits was $4(\mathrm{IQR}=2-6)$, the median time between study visits was 6.2 (IQR: 5.7-8.1) months, and the median follow up time per participant was 22.4 (IQR = 11.9-43.2) months. Baseline characteristics of the study sample are presented in Table 1. Over study follow-up, 97 (21 \%) injection initiation events were observed for an incidence density of 8.6 cases per 100 person years [95 \% Confidence Interval (CI): 7.010.6]. The median time to injection initiation from study enrolment was 11.2 months (IQR: 3.9-23.9), and the median number of years between initiation of noninjection "hard" drug use (defined as use of heroin, cocaine, crack, or crystal methamphetamine) and initiation of injection drug use was 7.1 (IQR = 4.6-9.5).

At some point during the study period 129 (28 \%) youth reported being unable to access addiction treatment. In total, 183 study observations included a report of being unable to access addiction treatment. Among these study observations, the most common type of addiction treatment that participants reported being unable to access was detox services $(n=76,41 \%)$, followed by treatment centers $(n=65,35 \%)$, recovery houses $(n=$ $20,10 \%)$, and counselors $(n=8,4 \%)$. The main reason participants reported being unable to access addition treatment was waiting lists $(n=118,66 \%)$, followed by logistical issues such as hours of optional, location, required paperwork etc. $(n=32,18 \%)$. Being rejected from the program for an unspecified reason $(n=16,9 \%)$, and having behavioral issues $(n=10,6 \%)$ were two other

Table 1 Baseline characteristics and Cox regression analysis for factors associated with injection initiation among street-involved youth $(n=462)$

\begin{tabular}{|c|c|c|c|c|c|c|}
\hline \multirow[t]{3}{*}{ Characteristic } & \multirow{2}{*}{\multicolumn{2}{|c|}{$\begin{array}{l}\text { Baseline Characteristics } \\
\text { Injection Initiation }\end{array}$}} & \multicolumn{4}{|c|}{ Bivariable and Multivariable Cox Regression Analysis } \\
\hline & & & \multirow{2}{*}{$\begin{array}{l}\text { Unadjusted } \mathrm{HR}^{\mathrm{a}} \\
(95 \% \mathrm{Cl})^{\mathrm{b}}\end{array}$} & \multirow[t]{2}{*}{$p$-value } & \multirow{2}{*}{$\begin{array}{l}\text { Adjusted HR } \\
(95 \% \mathrm{Cl})\end{array}$} & \multirow{2}{*}{$\begin{array}{l}p^{-} \\
\text {value }\end{array}$} \\
\hline & Yes $(n=97) n(\%)$ & $\mathrm{No}(n=365) \mathrm{n}(\%)$ & & & & \\
\hline \multicolumn{7}{|c|}{ Unable to access addiction treatment d,e } \\
\hline Yes & $15(15.5)$ & $33(9.0)$ & $2.19(1.27-3.78)$ & 0.005 & $2.02(1.12-3.62)$ & 0.019 \\
\hline No & $80(82.5)$ & $324(88.8)$ & & & & \\
\hline \multicolumn{7}{|c|}{ Years since initiated hard drug use (HR per additional year) } \\
\hline Median & 5.4 & 5.4 & $1.00(0.94-1.07)$ & 0.893 & $0.99(0.92-1.06)$ & 0.714 \\
\hline IQR & $(3.7-7.8)$ & $(3.1-7.9)$ & & & & \\
\hline \multicolumn{7}{|c|}{ Caucasian Ethnicity } \\
\hline Yes & $68(70.1)$ & $219(60.0)$ & $1.50(0.97-2.31)$ & 0.069 & $1.40(0.88-2.21)$ & 0.152 \\
\hline No & $29(29.9)$ & $146(40.0)$ & & & & \\
\hline \multicolumn{7}{|c|}{ Female Gender } \\
\hline Yes & $28(28.9)$ & $114(31.2)$ & $0.96(0.62-1.50)$ & 0.872 & $1.06(0.68-1.65)$ & 0.805 \\
\hline No & $69(71.1)$ & $251(68.8)$ & & & & \\
\hline \multicolumn{7}{|l|}{ Heroin Use $e^{c, d, e}$} \\
\hline Yes & $24(24.7)$ & $52(14.2)$ & $2.12(1.34-3.36)$ & 0.001 & $1.48(0.86-2.55)$ & 0.157 \\
\hline No & $70(72.2)$ & $307(84.1)$ & & & & \\
\hline \multicolumn{7}{|c|}{ Cocaine Use $e^{c, d, e}$} \\
\hline Yes & $43(44.3)$ & $186(51.0)$ & $1.17(0.77-1.78)$ & 0.449 & $1.06(0.69-1.64)$ & 0.782 \\
\hline No & $52(53.6)$ & $176(48.2)$ & & & & \\
\hline \multicolumn{7}{|c|}{ Crack Smoking ${ }^{\text {d,e }}$} \\
\hline Yes & $68(70.1)$ & $190(52.1)$ & $1.71(1.11-2.63)$ & 0.015 & $1.23(0.76-1.97)$ & 0.402 \\
\hline No & $27(27.8)$ & $171(46.8)$ & & & & \\
\hline \multicolumn{7}{|c|}{ Crystal Meth Use $e^{\mathrm{c}, \mathrm{d}, \mathrm{e}}$} \\
\hline Yes & $51(52.6)$ & $122(33.4)$ & $2.31(1.53-3.47)$ & $<0.001$ & $2.00(1.32-3.04)$ & 0.001 \\
\hline No & $43(44.3)$ & $238(65.2)$ & & & & \\
\hline
\end{tabular}

Not all cells add up to 462 as participants may choose not to answer sensitive questions

${ }^{\mathrm{a}} \mathrm{HR}$ hazard ratio; ${ }^{\mathrm{b}} \mathrm{Cl}$ confidence interval

cdenotes non-injection use; ${ }^{\mathrm{d}}$ denotes activities in the 6 months prior to follow-up interview; ${ }^{\mathrm{e}}$ refers to the activities lagged to the pervious available study follow-up; ${ }^{\mathrm{f}} p$-values based on Wald test 
common barriers. Note, out of the 183 observations that included a report of being unable to access addiction treatment, 10 observations did not specify the type of treatment that the participant was unable to access, 162 observations indicated one type of treatment, and $11 \mathrm{ob}-$ servations indicated 2 types of treatment. Similarly, 11 observations did not specify a reason the participant was unable to access treatment, 164 observations indicated one reason, and 8 observations indicated 2 reasons.

Table 1 shows the unadjusted and adjusted relative hazards of injection initiation. Being unable to access addiction treatment was significantly associated with injection initiation in both bivariable [hazard ratio $=2.19$, $95 \%$ CI: 1.27-3.78] and multivariable Cox regression analyses [adjusted hazard ratio $=2.02,95 \%$ CI: $1.12-3.62$ ].

\section{Discussion}

Among our sample of youth, $28 \%$ sought but were unable to access addiction treatment at some point during the study period. Youth who were unable to access addiction treatment were over two times more likely to subsequently initiate injection drug use, highlighting a critical missed opportunity to intervene to prevent injection initiation among high-risk youth. These findings are consistent with prior studies indicating that inability to access and engage with key health and social services, such as addiction treatment, housing, and employment negatively influences drug use behaviors and trajectories among vulnerable populations [10-12, 19, 23]. Prior cross-sectional analyses also found that contact with addiction treatment significantly delayed injection initiation among heroin users in the United States, highlighting the protective benefits of addiction treatment [24].

Given the importance of intervening early in youths' drug use trajectories to prevent injection initiation, our findings indicate that addressing deficiencies in youth addiction treatment, particularly with respect to waiting lists and logistical issues, should be a top priority. Numerous barriers to accessing addiction treatment have been identified in the literature and include: limited availability and insufficient use of evidence-based medication-assisted therapies; long wait times; lack of adequately trained providers; age restrictions; limited hours of operation; discrimination; and stigma, among others [18, 19, 25-31].

Our study has limitations. First, as with other studies of street-involved youth, the ARYS cohort is not a random sample and therefore these findings may not generalize to other populations. Second, this study is based on self-reported information and is susceptible to recall bias and socially desirable responding. We anticipate that any response bias would likely underestimate the prevalence of risk behaviors and therefore bias our results towards the null.
In summary, we found that inability to access addiction treatment predicted injection initiation among street-involved youth. Facilitating engagement with addiction treatment by reducing wait lists and increasing the availability of low-threshold evidence-based treatments offer important opportunities to engage with vulnerable youth and potentially prevent them from transitioning to injection drug use.

\section{Abbreviations \\ ARYS: At-Risk Youth Study; IQR: Interquartile range; Cl: Confidence Interval; HR: Hazard ratio.}

\section{Competing interests}

$\mathrm{JM}$ has received limited unrestricted funding, paid to his institution, from Abbvie, Bristol-Myers Squibb, Gilead Sciences, Janssen, Merck, and ViiV Healthcare. All other authors declare that they have no conflicts of interest.

\section{Authors' contributions \\ $\mathrm{KD}, \mathrm{TK}$, and EW designed the study and wrote the protocol, KD managed the literature search and prepared the first draft of the analysis; HD conducted the statistical analyses with input from KD and EW; all authors made a substantive intellectual contribution to the main content of the study, provided critical comments on the final draft, and approved the final manuscript.}

\section{Acknowledgements}

The authors thank the ARYS study participants for their contribution to the research, as well as current and past researchers and staff. We would specifically like to thank Cody Callon, Jennifer Matthews, Deborah Graham, Peter Vann, Steve Kain, Tricia Collingham, Kristie Starr and Carmen Rock for their research and administrative assistance. The study was supported by the US National Institutes of Health (U01DA038886). KD is supported by a MSFHR/St. Paul's Hospital-Providence Health Care Career Scholar Award and a Canadian Institutes of Health Research New Investigator Award. This research was undertaken, in part, thanks to funding from the Canada Research Chairs program through a Tier 1 Canada Research Chair in Inner City Medicine, which supports EW. JM is supported with grants paid to his institution by the British Columbia Ministry of Health and by the US National Institutes of Health (R01DA036307). Funding sources had no role in the: design and conduct of the study; collection, management, analysis, and interpretation of the data; and preparation, review, or approval of the manuscript; and decision to submit the manuscript for publication.

\section{Author details}

${ }^{1}$ British Columbia Centre for Excellence in HIV/AIDS, Vancouver, Canada. ${ }^{2}$ School of Public Policy, Simon Fraser University, Vancouver, Canada. ${ }^{3}$ Division of AIDS, Department of Medicine, University of British Columbia, Vancouver, Canada.

Received: 2 October 2015 Accepted: 23 December 2015 Published online: 06 January 2016

\section{References}

1. Miller CL, Kerr T, Strathdee SA, Li K, Wood E. Factors associated with premature mortality among young injection drug users in Vancouver. Harm Reduct J. 2007:4:1.

2. Miller CL, Wood E, Spittal PM, Li K, Frankish JC, Braitstein P, et al. The future face of coinfection: prevalence and incidence of HIV and hepatitis $C$ virus coinfection among young injection drug users. J Acquir Immune Defic Syndr. 2004;36(2):743-9.

3. Roy E, Haley N, Leclerc P, Sochanski B, Boudreau JF, Boivin JF. Mortality in a cohort of street youth in Montreal. Jama. 2004;292(5):569-74.

4. Werb D, Kerr T, Lai C, Montaner J, Wood E. Nonfatal overdose among a cohort of street-involved youth. J Adolesc Health. 2008;42(3):303-6.

5. Fuller CM, Vlahov D, Ompad DC, Shah N, Arria A, Strathdee SA. High-risk behaviors associated with transition from illicit non-injection to injection drug use among adolescent and young adult drug users: a case-control study. Drug Alcohol Depend. 2002;66(2):189-98. 
6. Debeck K, Kerr T, Marshall BD, Simo A, Montaner J, Wood E. Risk factors for progression to regular injection drug use among street-involved youth in a Canadian setting. Drug Alcohol Depend. 2013;133(2):468-72.

7. Baldwin P, Shrestha R, Potrepka J, Copenhaver M. The Age of Initiation of Drug Use and Sexual Behavior May Influence Subsequent HIV Risk Behavior: A Systematic Review. Isrn Aids. 2013;2013:976035.

8. Golub ET, Strathdee SA, Bailey SL, Hagan H, Latka MH, Hudson SM, et al. Distributive syringe sharing among young adult injection drug users in five U.S. cities. Drug Alcohol Depend. 2007;91 Suppl 1:S30-38.

9. Marshall BD, Kerr T, Qi J, Montaner JS, Wood E. Public injecting and HIV risk behaviour among street-involved youth. Drug and alcohol dependence. 2010;110(3):254-8.

10. Feng C, DeBeck K, Kerr T, Mathias S, Montaner J, Wood E. Homelessness independently predicts injection drug use initiation among street-involved youth in a Canadian setting. J Adolesc Health. 2013;52(4):499-501.

11. Richardson L, DeBeck K, Feng C, Kerr T, Wood E. Employment and risk of injection drug use initiation among street involved youth in Canadian setting. Prev Med. 2014;66:56-9.

12. Roy E, Haley N, Leclerc P, Cedras L, Blais L, Boivin JF. Drug injection among street youths in Montreal: predictors of initiation. J Urban Health. 2003;80(1):92-105.

13. Hadland SE, Werb D, Kerr T, Fu E, Wang H, Montaner JS, et al. Childhood sexual abuse and risk for initiating injection drug use: A prospective cohort study. Prev Med. 2012;55(5):500-4.

14. Werb D, Kerr T, Buxton J, Shoveller J, Richardson C, Montaner J, et al. Crystal methamphetamine and initiation of injection drug use among street-involved youth in a Canadian setting. CMAJ. 2013;185(18):1569-75.

15. Gerstein DR, Lewin LS. Treating drug problems. New England Journal of Medicine. 1990;323(12):844-8.

16. McCollister KE, French MT. The relative contribution of outcome domains in the total economic benefit of addiction interventions: a review of first findings. Addiction. 2003;98(12):1647-59.

17. Brands B, Leslie K, Catz-Biro L, Li S. Heroin use and barriers to treatment in street-involved youth. Addiction Research \& Theory. 2005;13(5):477-87.

18. Hadland SE, Kerr T, Li K, Montaner JS, Wood E. Access to drug and alcohol treatment among a cohort of street-involved youth. Drug Alcohol Depend. 2009;101(1-2):1-7.

19. Milloy MJ, Kerr T, Zhang R, Tyndall M, Montaner J, Wood E. Inability to access addiction treatment and risk of HIV infection among injection drug users recruited from a supervised injection facility. J Public Health (Oxf). 2010;32(3):342-9.

20. Phillips $\mathrm{M}$, DeBeck K, Desjarlais T, Morrison T, Feng C, Kerr T, et al. Inability to access addiction treatment among street-involved youth in a Canadian setting. Subst Use Misuse. 2014;49(10):1233-40.

21. Wood E, Stoltz JA, Montaner JS, Kerr T. Evaluating methamphetamine use and risks of injection initiation among street youth: the ARYS study. Harm Reduct J. 2006;3(1):18.

22. Kleinbaum DG, Klein M. Survival analysis. New York: Springer; 1996.

23. Steensma C, Boivin JF, Blais L, Roy E. Cessation of injecting drug use among street-based youth. J Urban Health. 2005;82(4):622-37.

24. Kelley MS, Chitwood DD. Effects of drug treatment for heroin sniffers: a protective factor against moving to injection? Soc Sci Med. 2004;58(10):2083-92.

25. Fiellin DA. Treatment of adolescent opioid dependence: no quick fix. JAMA. 2008:300(17):2057-9.

26. Garrett SB, Higa DH, Phares MM, Peterson PL, Wells EA, Baer JS. Homeless youths' perceptions of services and transitions to stable housing. Evaluation and program planning. 2008;31(4):436-44.

27. Greenfield BL, Owens MD, Ley D. Opioid use in Albuquerque. New Mexico: a needs assessment of recent changes and treatment availability Addiction science \& clinical practice. 2014:9:10

28. Hudson AL, Nyamathi A, Greengold B, Slagle A, Koniak-Griffin D, Khalilifard $F$, et al. Health-seeking challenges among homeless youth. Nursing research. 2010;59(3):212-8.

29. Kuehn BM. Medication helps make therapy work for teens addicted to prescription opioids. JAMA. 2010;303(23):2343-5.

30. Pecoraro A, Fishman M, Ma M, Piralishvili G, Woody GE. Pharmacologically assisted treatment of opioid-dependent youth. Paediatric drugs. 2013; 15(6):449-58.

31. Woody GE, Poole SA, Subramaniam G, Dugosh K, Bogenschutz M, Abbott P, et al. Extended vs short-term buprenorphine-naloxone for treatment of opioid-addicted youth: a randomized trial. JAMA. 2008;300(17):2003-11.

\section{Submit your next manuscript to BioMed Central and we will help you at every step:}

- We accept pre-submission inquiries

- Our selector tool helps you to find the most relevant journal

- We provide round the clock customer support

- Convenient online submission

- Thorough peer review

- Inclusion in PubMed and all major indexing services

- Maximum visibility for your research

Submit your manuscript at www.biomedcentral.com/submit
Biomed Central 